\title{
A abordagem da temática Energia no Curso de Ciências da Natureza - Licenciatura por meio da experimentação por investigação
}

\author{
El enfoque de la temática Energía en el Curso de Ciencias de la \\ Naturaleza - Licenciatura por medio de la experimentación por \\ investigación
}

\section{The approach of the theme Energy in the Course of Natural Sciences - Licenciatura through research experimentation}

\author{
Ticiane da Rosa Osório ${ }^{1}$
}

\begin{abstract}
Resumo
Nas últimas décadas o ensino de Ciências passou por diversas reformulações que visavam favorecer a aprendizagem e fomentar a educação científica. Emergiram também inúmeros recursos, como a Experimentação, que ganhou destaque a partir dos anos 70 nas aulas. Diante disso, este trabalho apresenta um recorte da pesquis a realizada no Mestrado Acadêmico em Ensino da Universidade Federal do Pampa - UNIPAMPA - Campus Bagé, na qual desenvolveu-se a Oficina intitulada "TIC e o ensino de Ciências da Natureza: Experimentação para a abordagem do tema Energia". Participaram 20 acadêmicos do segundo semestre do Curso de Ciências da Natureza - Licenciatura, UNIPAMPA - Campus Dom Pedrito. Objetivou-se desenvolver o conceito de Energia e suas transformações por meio da Experimentação por investigação. Caracteriza-se como uma pesquisa qualitativa, sendo os dados coletados através de um questionário semiestruturado. Resultados revelam que 14 (70\%) dos acadêmicos entenderam os objetivos das Experimentações. Reconheceram o caráter interdisciplinar nas abordagens ao destacarem mais de um componente curricular concomitantemente, ressaltando também as relações de Energia e suas transformações, como a Energia em forma de Calor. Por fim, considera-se relevante intervenções pedagógicas no contexto da formação inicial de professores, pois assim é possível explorar temas complexos como a Energia, potencializando a investigação, criticidade e reflexão.
\end{abstract}

Palavras-Chave: Formação inicial; Experimentação; Investigação; Oficina; Energia.

\section{Resumen}

En las últimas décadas la enseñanza de las Ciencias pasó por diversas reformulaciones que pretendían favorecer el aprendizaje y fomentar la educación científica. También surgieron innumerables recursos, como la Experimentación, que ganó destaque a partir de los años 70 en las clases. En este trabajo se presenta un recorte de la investigación realizada en el Máster Académico en Enseñanza de la Universidad Federal de Pampa UNIPAMPA - Campus Bagé, en la cual se desarrolló el Taller titulado "TIC y la enseñanza de Ciencias de la Naturaleza: Experimentación para el abordaje del enfoque tema de la energía ". Participaron 20 académicos del segundo semestre del Curso de Ciencias de la Naturaleza - Licenciatura, UNIPAMPA - Campus Dom Pedrito. Se pretendió desarrollar el concepto de Energía y sus transformaciones a través de la experimentación por investigación. Se caracteriza como una investigación cualitativa, siendo los datos recolectados a través de un cuestionario semiestructurado. Los resultados revelan que $14(70 \%)$ de los académicos entendieron los objetivos de las Experimentaciones. Reconocen el caratér interdisciplinario en los enfoques al destacar más de un componente curricular concomitantemente, resaltando también las relaciones de Energía y sus transformaciones, como la Energía en forma de Calor. Por último, se considera relevante intervenciones pedagógicas en el contexto de la formación inicial de profesores, pues así es posible explorar temas complejos como la Energía, potenciando la investigación, la criticidad y la reflexión.

\footnotetext{
${ }^{1}$ Licenciada em Ciências da Natureza; Universidade Federal do Pampa - UNIPAMPA; Bagé, Rio Grande do Sul, Brasil; ticiani_dp@hotmail.com
} 
Palabras claves: Formación inicial; Experimentación; Investigación; Taller; Energía.

\begin{abstract}
In the last decades the teaching of Sciences underwent several reformulations aimed at favoring learning and fostering scientific education. Numerous resources also emerged, such as Experimentation, which gained prominence in the 1970s in classrooms. Therefore, this work presents a study of the research carried out in the Academic Master's in Teaching of the Federal University of Pampa - UNIPAMPA - Campus Bagé, in which the Workshop entitled "ICT and the teaching of Natural Sciences: Experimentation for the Energy theme ". Participated 20 academics of the second semester of the Course of Natural Sciences - Bachelor, UNIPAMPA Campus Dom Pedrito. The objective was to develop the concept of Energy and its transformations through Research Experimentation. It is characterized as a qualitative research, being the data collected through a semistructured questionnaire. Results reveal that $14(70 \%)$ of the students understood the objectives of the Experiments. They recognized the interdisciplinary nature of the approaches by highlighting more than one curricular component concomitantly, emphasizing also the relations of Energy and its transformations, such as Energy in the form of Heat. Finally, it is considered relevant pedagogical interventions in the context of initial teacher training, because this way it is possible to explore complex themes such as Energy, enhancing research, criticism and reflection.
\end{abstract}

Keywords: Initial training; Experimentation; Research; Workshop; Energy.

\title{
1. Introdução
}

Há vários anos, o ensino de Ciências vem passando por reformulações referentes aos métodos de abordagem de conteúdos, conceitos e temas com o intuito de uma construção do conhecimento científico de forma significativa e que contemplasse a realidade dos estudantes. Ao longo dos últimos 50 anos algumas metodologias tomaram maior espaço na área do ensino de Ciências. Dentre as inovações metodológicas, destacam-se efetivamente a utilização da Experimentação e as atividades de práticas experimentais, a partir da década de 70, no contexto da sala de aula em todos os níveis de ensino (NARDI, 2005).

A Experimentação se constitui como uma estratégia pedagógica que quando bem desenvolvida pode promover uma construção do conhecimento contextualizada e significativa. Segundo as concepções de Oliveira (2010) a Experimentação contribui de diversas formas, tais como para atentar e despertar a atenção dos estudantes; motivar e desenvolver a criticidade; aprimorar a capacidade de observação e as habilidades manipulativas; aprender a analisar os resultados, conceitos científicos e o levante de hipóteses para os fenômenos observados; entre outras nas quais poder-se-ia elencar.

Vale destacar também que a Experimentação emerge como um recurso eficiente para o levantamento de problemáticas cotidianas estimulando a percepção de situações reais e contextualizadas por meio da investigação, análise e interpretação dos dados. A Experimentação também proporciona que o professor problematize e incentive a criticidade 
nos indivíduos, bem como o caráter investigativo e a curiosidade, renegando a simples transmissão do conhecimento científico (GUIMARÃES, 2009; JUNIOR et al., 2008).

Partindo do pressuposto e de acordo com as concepções supracitadas, este trabalho apresenta um recorte da pesquisa realizada no âmbito do Programa de Pós-Graduação Mestrado Acadêmico em Ensino da Universidade Federal do Pampa - UNIPAMPA Campus - Bagé. A pesquisa em andamento tem como objetivo geral analisar de que forma a Experimentação por investigação contribui para a aprendizagem significativa do tema Energia e suas transformações desenvolvidas por meio da Termoquímica.

Para tanto, nas investigações iniciais, elaborou-se uma oficina temática intitulada "TIC e o ensino de Ciências da Natureza: a utilização da Experimentação para a abordagem do tema Energia" que foi realizada com os acadêmicos do segundo semestre do Curso de Ciências da Natureza - Licenciatura (CNL) da UNIPAMPA, Campus - Dom Pedrito, no qual tencionou desenvolver o conceito de Energia e suas transformações por meio da Experimentação por investigação. A escolha pelo tema Energia se justifica pela necessidade de abordagens que facilitem seu entendimento, já que é considerado complexo e abstrato para muitos pesquisadores.

Na visão de Barbosa e Borges (2006) é considerado como um dos mais abstratos e complexos de ser entendido dentro do currículo escolar. Devido a esta complexidade, o estudante em grande parte das vezes confunde este conceito com outros comumente relacionados à potência, movimento e força. Acredita-se que esta abstração justifique-se pela diversidade de representações que a Energia pode ser encontrada, tais como a transferência de calor entre os corpos, no calor de uma chama, na luz natural solar, na condução da eletricidade, nos movimentos em geral, dentre outros exemplos (GASPAR, 2009).

Por este motivo, e tendo conhecimento da importância das relações entre os componentes curriculares da área das Ciências da Natureza, os Parâmetros Curriculares Nacionais $(\mathrm{PCN})$ expressam que

A energia é um exemplo importante de um conceito comum às distintas ciências, instrumento essencial para descrever regularidades da natureza e para aplicações tecnológicas. Na Física, pode ser apresentada em termos do trabalho mecânico necessário para impelir ou para erguer objetos, quando se calcula a energia cinética do movimento de um projétil ou veículo, ou a energia potencial da água numa barragem. [...] A falta de unificação entre os conceitos de energia pode resultar em uma "colcha de retalhos energética", a ser memorizada, das energias mecânica e térmica, luminosa, sonora, química, nuclear e tantos outros adjetivos, alguns pertinentes, outros não. Na Biologia e na Química, as energias não são menos importantes e nem menos variadas em suas designações e, no fundo, se trata da mesma energia da Física. [...] É preciso um esforço consciente dos professores das três disciplinas para que o aluno não tenha de fazer sozinho a tradução dos discursos 
disciplinares ou, o que é pior, concluir que uma energia não tem nada a ver com a outra (BRASIL, 2002, p.29).

Conforme o excerto acima destacado percebe-se a importância da interação entre os conceitos estabelecidos na Química, Física e Biologia para o ensino do tema Energia, pois, por muitas vezes os estudantes podem ter dificuldade em reconhecer estes aspectos interdisciplinares.

\section{Contexto da Pesquisa}

A Universidade Federal do Pampa (UNIPAMPA) é uma Instituição Superior multicampi que esta distribuída em 10 cidades do estado do Rio Grande do Sul (Alegrete, Bagé, Caçapava do Sul, Dom Pedrito, Itaqui, Jaguarão, São Gabriel, São Borja, Santana do Livramento e Uruguaiana). A Figura a seguir sinaliza, no mapa do Rio Grande do Sul, os estados nos quais a UNIPAMPA esta presente.

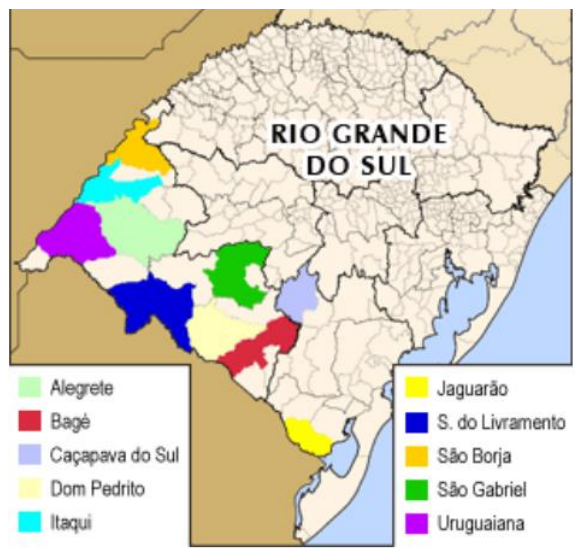

Figura 1: Abrangência geográfica da Instituição no estado.

Fonte: porteiras unipampa.edu.br/dompedrito

O lócus no qual ocorreu a intervenção deste trabalho foi na UNIPAMPA - Campus Dom Pedrito, tendo como sujeitos os acadêmicos do segundo semestre do Curso de Licenciatura em Ciências da Natureza. Este Campus localiza-se na microrregião da Campanha, sendo o $4^{\circ}$ maior município em extensão do Rio Grande do Sul, com $5.250 \mathrm{~km}^{2}$ abrangendo uma população de aproximadamente 38.916 .

O Curso de Ciências da Natureza - Licenciatura é constituído pela formação científica interdisciplinar e profissional, ética e reflexiva, comprometida com o desenvolvimento humano e a sustentabilidade ambiental. Além disso, o Curso tem como principal objetivo à formação de professores na área de Ciências da Natureza, ou seja, o mesmo habilita o 
licenciado ao Ensino de Ciências, Biologia, Física e Química nas séries finais do Ensino Fundamental e Médio (UNIPAMPA, 2015).

\section{Caminhos da pesquisa}

Esta pesquisa pode ser classificada predominantemente como qualitativa, no entanto, também utiliza dados que podem ser quantificados para sua complementação.

Gil (2010, p. 133) especifica que de modo geral as pesquisas classificadas como qualitativas são entendidas como menos formalizadas que as quantitativas, já que possibilitam maior flexibilidade e forma simplificada organizacional. No excerto abaixo, o autor ainda argumenta ainda que esta

[...] depende de muitos fatores, tais como a natureza dos dados coletados, a extensão da amostra, os instrumentos de pesquisa e os pressupostos teóricos que nortearam a investigação. Assim, define-se essa sistematização envolvendo ampliação, interpretação, aprofundamento, sequenciamento e categorização dos dados .

$\mathrm{O}$ autor ainda especifica que nas pesquisas qualitativas "[...] o conjunto inicial de categorias em geral é reexaminado e modificado sucessivamente, com vista em obter ideais mais abrangentes e significativas", levando em consideração todos os aspectos que possam enriquecer os resultados encontrados como esquemas, imagens, símbolos e narrativas (GIL, 2010, p. 134).

Além disso, a pesquisa qualitativa também possibilita ao pesquisador uma profunda imersão referente ao tema estudado, explorado e investigado, considerando que sua atuação é essencial para os dados resultantes (CHIZZOTTI, 2008).

A Oficina temática foi estruturada em seis momentos específicos. No primeiro momento, os proponentes realizaram a explanação por meio de slides informativos nos quais conceituaram o que é a Experimentação e diferenciaram os tipos de Experimentações que podem ser desenvolvidas no contexto de sala de aula (demonstrativa, verificativa e investigativa).

No segundo momento, foi realizada a Experimentação nomeada como "Batata oxigenada", no qual lançou a questão problema "Por que o peróxido de hidrogênio sofreu alteração no seu estado físico e químico ao entrar em contato com a batata?’. No Quadro 1 estão dispostos os materiais disponibilizados para que os acadêmicos realizassem a Experimentação.

- Rodelas médias de batata inglesa;

- Placas de petri;

- Béqueres;

- Água oxigenada v. 20 e 30; 
No terceiro momento, a Experimentação desenvolvida foi a intitulada como "Fígado espumante", por meio do questionamento "Por que o fígado bovino espuma ao entrar em contato com o peróxido de hidrogênio?”. No Quadro 2 estão dispostos os materiais disponibilizados para que os acadêmicos realizassem a Experimentação.

\section{MATERIAIS}

- Porções de fígado bovino cru e cozido;

- Placas de petri;

- Béqueres;

- Água oxigenada v. 20 e 30;

- Luvas de silicone;

Quadro 2: Materiais utilizados na Experimentação

Fonte: Autora (2018)

Ressalta-se que a Experimentação do "Fígado espumante" baseou-se na Experimentação da "Batata oxigenada", pois além da Energia como enfoque principal da oficina temática, optou-se também por realizar a comparação entre as células vegetais e as animais em contato com o peróxido de hidrogênio. No quarto momento, realizou-se a Experimentação nomeada como "Condução de calor nos metais", assim, a questão norteadora foi "Por que os metais são considerados bons condutores de calor?”. Nesta Experimentação utilizou-se barras de alumínio e de cobre, água quente, água gelada e recipiente plástico.

No quinto momento, a Experimentação desenvolvida foi a do "Calorímetro de água", tendo como questionamento norteador "Qual a quantidade de Energia que as sementes de amendoim, nozes e castanhas podem fornecer ao organismo humano?”. O Quadro 3 expõe os materiais que foram utilizados para a realização desta Experimentação.

- 1 haste de titulação;

MATERIAIS

- 1 isqueiro ou fósforo;

- 100 ml de água;

- fita adesiva larga;

- 1 clip grande;

- 1 termômetro;

- 1 Erlenmeyer;

- Nozes, castanhas e amendoins.

Quadro 3: Materiais utilizados para a Experimentação

Fonte: Autores (2018)

Por fim, como última Experimentação realizou-se a "Condução de eletricidade por substâncias químicas", o problema lançado foi "Como algumas substâncias podem conduzir eletricidade e outras não?”. Utilizou-se um circuito de Energia simples (sirene), Becker, 
limão, vinagre, açúcar, suco de laranja concentrado, sal e água. Foram mergulhados os dois fios do circuito elétrico em cada uma das substâncias em meio aquoso. Aquelas que conduziram a eletricidade acenderam a lâmpada com intensidades diferentes.

Com o intuito de verificar quais as aprendizagens os acadêmicos haviam construído ao longo da oficina temática, como finalização da intervenção aplicou-se um instrumento de coleta de dados. O mesmo foi estruturado em formato de quadro, e em cada uma das Experimentações foi questionado: 1) Qual o objetivo da Experimentação?; 2) Qual(is) o(s) componente(s) curricular(es) foram abordado(s)?; 3) Esta Experimentação pode ser realizada em qualquer espaço de aprendizagem? Por quê?; 4) Qual a relação da Experimentação com o tema Energia? De que modo se apresenta?; 5) O que entendi sobre esta Experimentação?

\section{Resultados e discussões}

A pesquisa foi realizada com os acadêmicos do segundo semestre da CNL, turma composta por 20 sujeitos (14 mulheres e seis homens) com idades compreendidas entre $17 \mathrm{e}$ 44 anos.

Na primeira questão do instrumento de coleta de dados, percebeu-se que grande parte dos acadêmicos 14 (70\%) identificaram os objetivos da realização de cada Experimentação, predominando os verbos identificar, observar e verificar. A primeira Experimentação e a segunda possuíam objetivos semelhantes, no entanto a "Batata oxigenada" teve uma maior exploração e contextualização voltada para as células vegetais, ao passo que, na Experimentação "Fígado espumante" a abordagem relacionou-se mais especificamente a célula animal.

$\mathrm{Na}$ análise da segunda questão, verificou-se que os acadêmicos identificam ligações de duas ou mais componentes curriculares da área das Ciências da Natureza. Como exemplo, destaca-se, o resultado da primeira Experimentação, já que 13 (65\%) reconheceram as relações entre Química, Física e Biologia; quatro (20\%) mencionaram Química e Física; e três $(15 \%)$ identificaram ligações entre Biologia e Química. Este fato comprova que desde o inicio da formação inicial, e justamente pelo perfil diferenciado do Curso, os acadêmicos são levados a realizarem as interligações interdisciplinares para abordagens de temáticas abstratas, neste caso a Energia.

No que se refere à terceira questão cabe destacar que, todas as Experimentações realizadas na oficina temática apresentadas neste trabalho, podem ser desenvolvidas em qualquer espaço de aprendizagem, já que os materiais utilizados em cada uma podem ser adaptados por outros de baixo custo. No entanto, cabe ao professor atentar-se as precauções 
necessárias a fim de zelar pela segurança dos sujeitos envolvidos. E este foi um dos aspectos relatados por alguns acadêmicos, pois em suas respostas demonstram entendimento de que todas as cinco Experimentações podem ser realizadas em diversos contextos de aprendizagem, sendo estes formais ou não. No entanto, destacaram que o risco sempre está presente de uma forma ou outra, e que o professor deve estar atento para que a periculosidade seja a menor possível.

$\mathrm{Na}$ quarta questão, verificou-se que 14 (70\%) dos acadêmicos entenderam a relação do tema Energia com as Experimentações desenvolvidas. Na primeira e segunda Experimentação todos relacionaram de forma correta ao mencionarem como respostas: Cinética e catálise das reações químicas; Para acelerar a enzima catalase e Energia ativada e aplicada para catalisar a batata. Na Experimentação "Condução de calor nos metais", os acadêmicos expressaram em suas repostas que o Calor é uma forma de Energia, e que dessa maneira a relação estabelecida era justamente demonstrar que o Calor é uma Energia em trânsito.

Já na Experimentação denominada como "Calorímetro de água" 15 (75\%) dos acadêmicos entenderam que a relação estabelecida entre a temática e a Experimentação eram as transformações de Energia que ocorreram na combustão das sementes, ou seja, a Energia química e a Energia térmica. Além disso, alguns ainda destacaram em suas respostas que no momento da combustão houve perca de Energia para o meio e que dessa forma a Energia é transformada, mas que dependendo desta transformação pode ocorrer uma perca considerável para o meio. De modo semelhante, na última Experimentação mencionaram que determinadas substâncias quando entram em contato com meio aquoso podem gerar eletricidade, tais como limão e sal.

Em análise da última questão diagnosticou-se a oficina temática atrelada à utilização da Experimentação por investigação proporcionou aos acadêmicos o entendimento das relações entre Energia, suas transformações e o cotidiano, facilitando assim as conexões necessárias para a aprendizagem da temática em voga, que por vezes é vista como abstrata e distante da realidade.

Este fato se comprova ao verificar que nas respostas os acadêmicos destacam os conceitos científicos explorados como no caso da primeira e segunda Experimentação que envolvia o processo de aceleração da enzima catalase nas reações químicas. Assim como na terceira Experimentação, onde destacaram que o Calor é uma forma de Energia, exemplificando também que dependendo do metal a condução pode ser mais intensa, e, ainda argumentaram que determinados metais, como o cobre e o alumínio, são excelentes condutores de Energia elétrica. 
Das aprendizagens constituídas na quarta Experimentação, percebeu-se que os participantes associaram a combustão das sementes com a produção de Energia para o metabolismo humano, ao mencionarem a transformação de Energia química em Energia térmica. Argumentaram ainda que os processos químicos envolvidos no metabolismo humano são bem mais complexos que a forma apresentada na Experimentação, e que a perca de Energia para o meio foi muito mais considerável do que no processo digestivo humano. $\mathrm{Na}$ última Experimentação, diagnosticou-se que os acadêmicos relacionaram que algumas substâncias, como o limão, quando em contato com meio aquoso dissociam gerando íons positivos e negativos, sendo estes capazes de conduzir eletricidade.

\section{Considerações finais}

Sendo a Energia admitida como um dos conceitos mais complexos dentro da área de Ciências da Natureza, cabe aos professores pensarem em alternativas que facilitem o entendimento desta temática, por meio de metodologias que promovam uma conexão entre teoria e prática. Além disso, torna-se indispensável à utilização de estratégias de ensino que envolva os estudantes de forma que os mesmos tenham a possibilidade de interagir, investigar e explorar os conceitos desenvolvidos.

Dessa maneira, por meio da realização desta Oficina temática, verificou-se que a maioria dos acadêmicos entendeu os objetivos estimados para cada Experimentação e assim relacionaram também as interligações interdisciplinares dos componentes curriculares que compõem a área das Ciências da Natureza. Pode-se afirmar este aspecto diante da análise dos resultados encontrados na segunda questão, aonde reconheceram mais de uma disciplina envolvida na abordagem do tema Energia.

Outro resultado que merece ser ressaltado, se refere às aprendizagens pelos participantes mencionados na quinta questão. Como exemplo pode-se citar as menções em que os mesmos identificaram a Energia e suas transformações, o Calor como uma forma de Energia e a dissolução de íons positivos e negativos em meio aquoso para gerar eletricidade através de diferentes substâncias, especialmente o limão e o sal.

Por fim, considera-se relevante a utilização da Experimentação por investigação para a abordagem de temáticas como a Energia no contexto da formação inicial de professores, já que por meio deste tipo de exploração conceitual, os acadêmicos podem ter maiores possibilidades de realizarem suas futuras aulas de forma diferenciada e com maior dinamismo. 
Pretende-se seguir com as investigações acerca de metodologias que promovam um entendimento esclarecido e interdisciplinar em relação à Energia e suas transformações com o intuito de contribuir para uma aprendizagem significativa e contextualizada da temática em questão. Este trabalho tem apoio financeiro da FAPERGS.

\section{Referências}

BARBOSA, J. P. V; BORGES, A. T. O entendimento dos estudantes sobre energia no início do ensino médio. Caderno Brasileiro de Ensino de Física. v. 23, n. 2: p. 182-217, 2006. ISSN: 2175-7941. Disponível em: https://periodicos.ufsc.br/index.php/fisica/article/view/6275. Acesso em: 14 jan. 2019.

BRASIL. Ciências da Natureza, Matemática e suas Tecnologias. Parâmetros Curriculares Nacionais - Ensino Médio: Orientações Educacionais Complementares aos parâmetros Curriculares Nacionais. Brasilia: MEC/SEMTEC, 2002.

CHIZZOTTI, A. Pesquisa qualitativa em ciências humanas e sociais. Petrópolis- RJ: Vozes, 2008.

GASPAR, A. Experiências de Ciências. São Paulo: Ed. Ática, 2009.

GIL, A. C. Como elaborar projetos de pesquisa. 5. ed. São Paulo: Atlas, 2010.

GUIMARÃES, C. G. Experimentação no Ensino de Química: Caminhos e Descaminhos Rumo à Aprendizagem Significativa. Química Nova na Escola, v. 31, n. 3, p. 198-202, 2009. ISSN: 2175-2599.

Disponível em: http://webeduc.mec.gov.br/portaldoprofessor/quimica/sbq/QNEsc31_3/08-RSA-4107.pdf. Acesso em: 14 jan. 2019.

NARDI, R. A área de Ensino de Ciências no Brasil: fatores que determinaram sua constituição e suas características segundo pesquisadores brasileiros. 2005. 166 f. Tese (Doutorado em Ensino) - Faculdade de Ciências, Bauru, 2005.

OLIVEIRA, J. R. S. Contribuições e abordagens das atividades experimentais no ensino de ciências: Reunindo elementos para a prática docente. Acta Scientiae, Canoas, v. 12, n. 1, p. 139-153, jan./jun., 2010. ISSN: 2317-8957. Disponível em: http://w3.ufsm.br/laequi/wpcontent/uploads/2015/03/contribui\%C3\%A7\%C3\%B5es-e-abordagens-de-atividadesexperimentais.pdf. Acesso em: 14 jan. 2019.

UNIPAMPA - Universidade Federal do Pampa - Projeto pedagógico de curso de Licenciatura em Ciências da Natureza, 2015. 2 Disponível em: http://dspace.unipampa.edu.br/bitstream/riu/110/1/PPC_Ci\%C3\%AAncias\%20da\%20Naturez a_Dom\%20Pedrito.pdf> Acesso em 03 de nov. de 2017. 